Indian J Anim Health (2021), 60(2): 303-308

DOI: https://doi.org/10.36062/ijah.2021.12721

\title{
Heat and touch sensation received Nobel Prize nomination in medicine: detail to be defined
}

\author{
Tapas Goswami ${ }^{*}$
}

${ }^{1}$ Emeritus Scientist, Immunology Section, Indian Veterinary Research Institute, Izatnagar - 243122 , Uttar Pradesh, India

\section{Perception of sensation}

We might have experienced the warmth of the sun while walking barefoot across a field in midday summer, the chilliness of the morning breeze of winter, and the feeling of the soft texture of blades of grass covered with dewdrops underneath the feet. Somehow, we can perceive the changes in environmental temperature; therefore, we exhibit appropriate behavioural responses such as wearing a coat during chilly weather, preferring to use a potholder to grab a hot frying pan, and never handling dry-ice without gloves in our laboratory. These behavioural responses are due to the thermosensation mechanism operating in our bodies. How we perceive and interpret all these warmth, coldness, soft-touch that come from the physical world around us through our sense organs has been of interest to mankind for since long. Have we ever imagined that there is a science-based hidden mystery behind this ubiquitous experience towards our nature? But eminent researchers David Julius and Ardem Patapoutian are two distinguishing individuals who have disclosed the mechanism of conversion of physical stimuli into electrical impulse within nerve cells, crowned them to receive Nobel Prize in Medicine for the year 2021.

\section{About the winners}

Russian-immigrant David Julius, an American citizen, was born on $7^{\text {th }}$ November 1955 , in Brighton Beach, Brooklyn, where he completed his primary and secondary education and graduated from the Massachusetts Institute

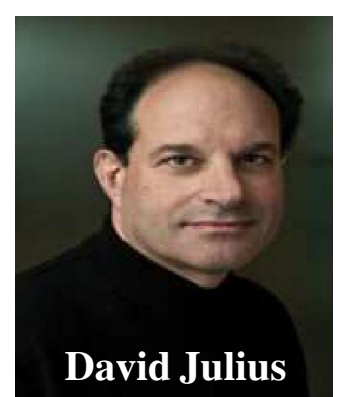

of Technology with a degree in Life Science in the year 1977. In 1984 he pursued post-doctoral work at Columbia University with the neurobiologist Richard Axel a Nobel laureate of 2004. He is currently occupying the chair of the department of physiology at the School of Medicine, University of California, San Francisco.

Another researcher, Ardem Patapoutian, an Armenian born and grew up in Lebanon before coming to the US. He studied at Beirut University for a year before moving to the United States in 1986 and later became a US citizen. He received his bachelor's degree in cellular and developmental biology from the University of California, Los Angeles, in 1990. His current field of research is to probe how mechanical stimuli are converted into chemical signals at the Doris

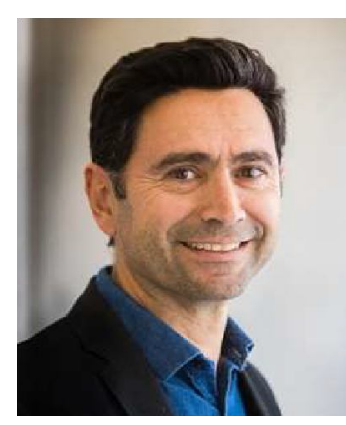

Ardem

Patapoutian Neuroscience Centre, La Jolla Scripts Research Centre.

\section{History of discovery}

During the 1700 s, the philosopher René Descartes predicted some connecting threads or tube-like structures present in different skin

Corresponding Author, E Mail: goswami.tapas@gmail.com 
parts that connect to the brain. According to him moving particles of fire on the skin pull the thread and open the valve in the brain; concurrently, he predicted that any part of the body would send a signal to the brain as soon as it came in contact with fire (DeLeo, 2006; Moayedi and Davis, 2013). Since ancient times the Greek physician Claudius Galen, most celebrated anatomist and practised physician in the Roman Empire Marcus Aurelius (161-180 $\mathrm{CE})$, proposed that the external and internal organs of the human body communicate with each other through the nervous system. As per his theory, sensations, including pain, were mediated by nerves connecting the organs to the brain (Stranding, 2016). Although the current scientific view does not accept the above theory, there are some analogies with the neurotransmitter mechanism operating in our body. During the past century, scientists discovered specialized sensory neurons, which have long processes called nerves, located through our body; in some way, the nerves must convert the physical stimuli of heat and touch into a biological signal.

\section{Notation or negation of sensation}

When we taste a green chilli, we describe it as hot; similarly, if we touch a boiling cup of water, our sense organ feels it as hot; the elucidation is not just a figment of our imagination. The hotness of the chilli and hot water pot is not similar. Irrefutably there is a difference in hotness while describing these two completely unrelated physical objects. Yet, the sensations of extreme spiciness and burning heat are intertwined in our nervous system at a molecular level. A chemical ingredient in chilli known as capsaicin has already been described as a cause of inflammation which gives a pungent taste, which we invariably describe as the hotness of chilli (Wood et al., 1988). According to David Julius, some kinds of organic sensors are precisely located at the terminal part of the nerve ending to sense the capsaicin present in chilli. He also opined that if for some reason or other this capsaicin comes in contact with an organic sensor, a message in the form of an electrical signal is transmitted immediately through the nerve cells to the brain resulting initiation of a chemical reaction to set in the end results escalate the body temperature. Another experiment performed independently by Ardem has established a novel class of biosensors sensitive to force or pressure acting on the body from an external source in the form of touch. This new biological sensor (receptor) is sensitive to mechanical stimuli in the skin and internal organs too.

Further research has shown that sensitive nerve cells (neurons) react accordingly by assessing the physical changes taking place in our environment (Peier et al., 2002). Much earlier to it, Joseph Erlanger and Herbert Gasser discovered the involvement of two religiously different sensitive nervous systems in the field of medicine for painful touch, and soothing touch differs in their performance. But the fundamental question of how temperature differences and mechanical energy are converted into an electrical impulse in the nervous system has not been answered until the findings of David Julius and Ardem Patapoutian brought it to light. Ardem first discovered a specific gene present in a different type of cells, which has been proven to be entirely responsible for sensing external force and pressure in the form of touch; simply, we can say touch-sensitive receptor coding gene identification. When he managed to silence a particular gene, the cells which were earlier sensitive to these external stimuli lost their "sensitivity" and became insensitive (Coste et al., 2010). Subsequently, he initiated to silence a total of 72 genes one after another; but despite successively silencing 71 genes, no success was achieved. Fortunately, silencing the $72^{\text {nd }}$ gene yielded spectacular results making the cell insensitive to external stimuli. The ability to experience the outside world and respond positively to changing environments is essential for animal survival. The somatosensory system also provides information about the movement of the organs of the human body, such as 
proprioception, touch, and temperature sensation. Harmful stimuli, like mechanical stress and painful heat, can stimulate a multifaceted nervous system. Accurately estimating the mechanical energy generated by touch, no matter how small it is, our cell receptors can recognize the source of the physical stimuli, structure, and shape of the object from which it has originated. The task of determining the qualitative difference of vibrations generated by touch is performed by different types of sensory neurons.

\section{David Julius was curious}

In 1990 David Julius, while working with the serotonin receptor family, switched to find functional markers for somatosensory neurons that are involved in pain sensation using capsaicin, a chemical compound found in chilli. The active ingredient capsaicin (8-methyl-nvanillyl-6-nannamide, 8-methyl-N-vanillyl-6nonenamide) belongs to the vanilloid type of compounds. These compounds have a vanillyl group having a ring structure. Significant quantities of capsaicin are present in fruits of plants of the genus Capsicum. The discovery of capsaicin dates back to the $16^{\text {th }}$ century. Research on these chemicals initiated by David Julius has paved the way for the discovery of heat-sensitive receptors in the body. Research findings have shown that as soon as the chemical comes in contact with the mouth or lips, it starts a hot sensation; thereby, we start sweating. Thus it seems that capsaicin can buzz the brain into thinking there is an actual change in body temperature. The process by which the sweat secretes is different from the sweat produced from the body during physical exercise. Capsaicin is responsible for stimulating sensitive nerves; as a result, ion channels are opened for entry and exit of ions to intracellular location. Living cells always remain in an aqueous environment saturated with soluble ions. The ion channels are the gateway through which ions get into intracellular location. The ion channels are not remained open throughout for the movement of chemical ions. As per the cell-specific requirements, this route is open only for a transitory period, and immediate closure is ensured once the job is over. Physical information about heat and touch stimulates a specific gene fragment stored in the nerve cell to open the ion channel. In his laboratory at the University of California, San Francisco, David Julius analyzed the genetic sequence of nerve cells of mice exhibiting sensitivity towards capsaicin. From the genomic DNA of dorsal root ganglia of mice, he artificially synthesized millions of complementary DNA (cDNA) fragments corresponding to the genes that are active in sensory neurons hoping that at least one of these would contain the gene for the capsaicin receptors (Caterina et al., 1997).

In the next step, he selected another mouse cell that was insensitive and refractory towards capsaicin as a model in his experiment. From the cDNA library collection, he introduced single DNA fragments into the cells one at a time in a sequential manner so that capsaicin insensitive cells received a single cDNA fragment in a given period to express the gene function of implanted DNA. He then exposed those transgenic cells to capsaicin to record their sensory response. In this process, after screening a large number of DNA fragments, the team finally succeeded in identifying that the insertion of a typical cDNA fragment could restore the sensitivity towards capsaicin. The identified gene fragments (which can reinstate cells sensitive) were turned out to code for a novel protein named TRPV1, an integral structural protein of capsaicin receptor localized in the cell's outer membrane (Fig. 1). The TRPV1 (pronounced trip, Transient receptor potential cation channel subfamily $\mathrm{V}$ member 1) functionally behaves as an ion channel indeed. The naming justifies its function: one of the members of protein sub-family number five having ion channel function for a transitory period acts as a receptor. The gene responsible for the synthesis of TRPV1 is written in italic form Trpv1.The primary goal of Julius David's was to find a receptor that was sensitive to 


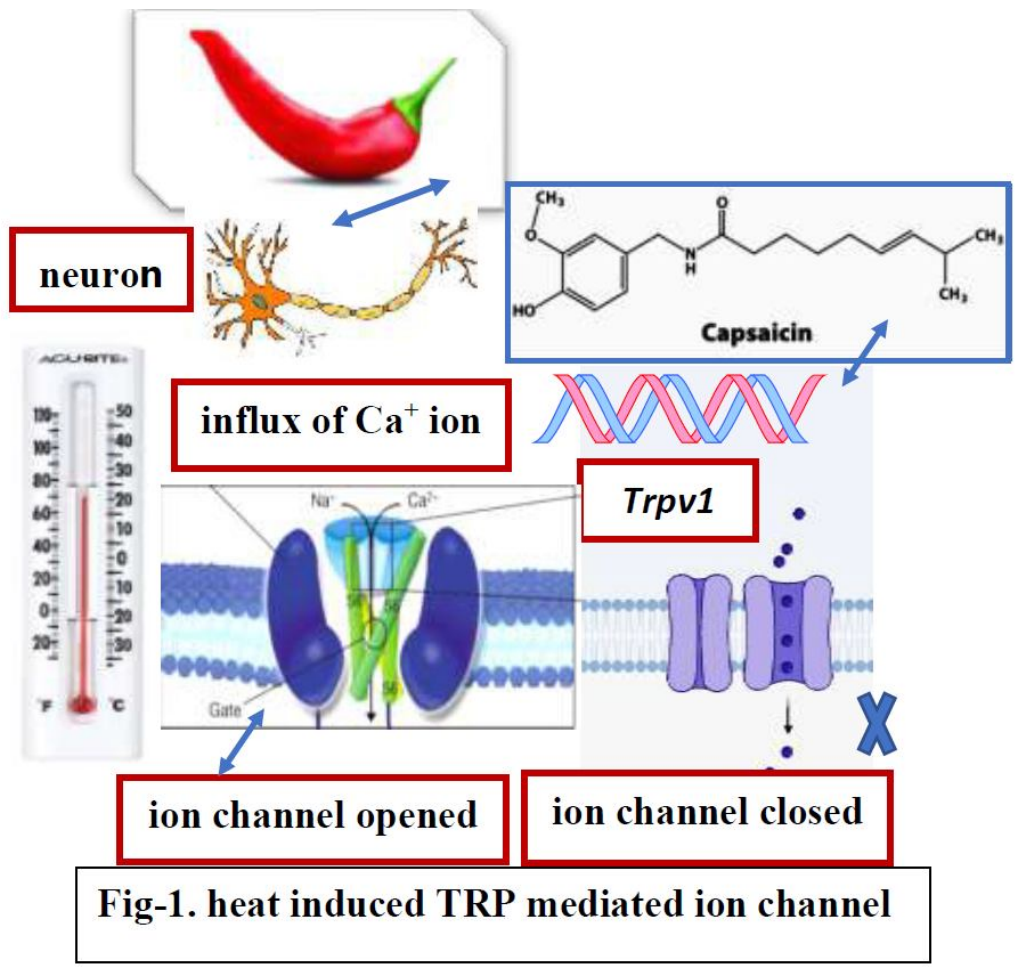

to sense the temperature variation of the surrounding environment. The activation of this receptor opens up the ion channel to accelerate the influx of calcium ions for intracellular location. The topology and the architecture of subunits of TRP channel proteins are similar to that of sodium and potassium channel. Information retrieved from structural biology has provided insight into ligand recognition, ion permeation, and gating. The binding of capsaicin can cause a conformational change in these proteins; thereby, the ion channel gate opens up. Nerve cells, once exposed to noxious heat above $40^{\circ} \mathrm{C}$, can sense the temperature variation; thereby, activation of Trpvl gene takes capsaicin, but he stretched further to find out whether the newly identified receptor was sensitive towards heat or not; if yes, then what is the thresh hold limit of this receptor? $\mathrm{He}$ demonstrated that capsaicin binds to a receptor called TRPV1, which is expressed by cells of the central nervous system, and once it binds, it triggers a severe burning sensation. We know that all the cells in our body are studded with several complex protein structures, out of which few are functionally behave as ion channels. These proteins are porous and produce minute electric fields that allow some ions to pass through their pores and in quantities determined by the cells' needs. Julius established that binding capsaicin to TRPV1 allows intracellular influx of positively charged calcium ions, producing a potential difference across the cell membrane, resulting in an electric signal that flows through conducting neurons until it reaches the brain. When Julius tested how TRPV1 reacted to warm temperature, he noticed that heat opened the channel for ions. While working with capsaicin, eventually, Julius came to know that he had discovered a heat-sensitive receptor. This receptor is physiologically active place for safety. A plant compound menthol, when applied to the skin, can give a soothing coolness; this may be due to the binding of this menthol compound to some other different receptor, speculated by David Julius. David Julius and Patapoutian working independently observed that cold sensation in humans and mice starts around $28^{\circ} \mathrm{C}$, and human skin can detect a difference of $0.5^{\circ} \mathrm{C}$ temperature with precision, whereas the precision limit for warmth detection thresholds of around $1^{\circ} \mathrm{C}$ for humans was recorded (Stevens and Choo, 1998).

Ultimately another temperature receptor on the cells, which is sensitive to cold rather than heat, was discovered by them and named TRPV8. It indicates that humans can recognize temperature differences through two different sensory systems, one for warmth and one for cold, and governed by distinctive genetic elements. This has paved the way for the identification of TRPM2, another member under the TRP channel family, governing the temperature sensation in man and mice. Deletion of the TRMP2 gene in mice resulted in deficits in the sensation of innocuous warm 
temperatures in the range $\left(33-38^{\circ} \mathrm{C}\right)$. Discrimination between warm and cool temperatures depends on the simultaneous activation of warmth-sensing and inhibition of cold-sensing nerve fibres. Similarly, the deletion of the TRMP8 gene in mice made them insensitive to nonlethal cold. A third TRP channel TRPA1 is responsible for the detection of a wide variety of active chemical compounds in mustard oil, horseradish, cinnamon, garlic, cloves and ginger, as well as lipid compounds, environmental irritants, and other chemicals.

\section{How much touch is too much?}

Even after unfolding the chapter on the heat-sensitive receptor, it was still unclear how the application of external force or pressure is converted to touch sensation. It was already known that the microbes Escherichia coli carry specific structures that are sensitive to detect external force or pressure of the surrounding environment, but identification of such receptors in vertebrates was out of their reach (Martinac et al., 1987). Ardem Patapoutian, following an innovative technique, identified mechanosensitive cell lines called Neuro 2A that can generate a measurable amount of electrical current across the plasma membrane when subject to external pressure force through touch. He speculated that the electrical signal generation is due to the presence of a receptor functionally acting like an ion channel (Fig. 2). In the next step, with the rigorous experiment, he could establish that the activation of receptors due to external pressure was governed by a single gene out of 42 genes screened during their investigation.

\section{Sensor for pressure}

Employing RNA interference technique total of 72 candidate genes were sequentially silenced one-by-one, and the quantum of electrical current was measured through patchclamp technique, ultimately confirming that a particular gene once silenced the sensory cells

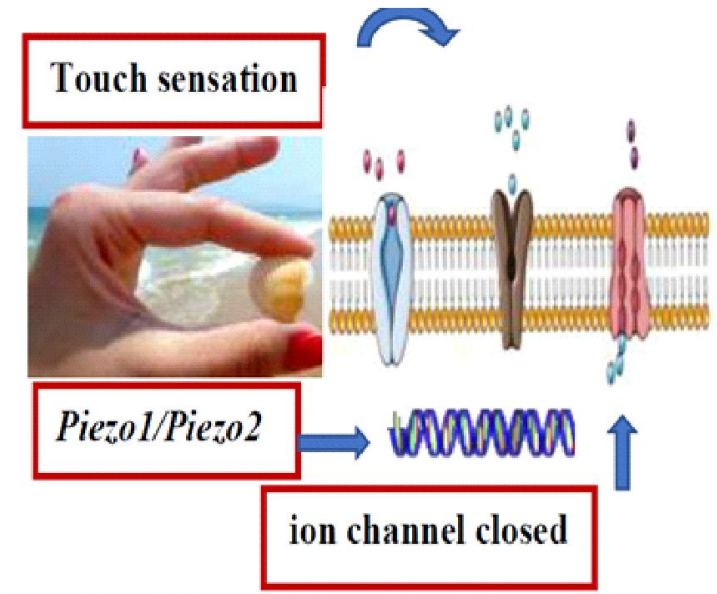

Fig-2. Pressure sensitive PIEZO-1/PIEZO-2 ion channel

lost their sensitivity towards external force and became insensitive towards external mechanical energy. The loss of sensitivity is controlled by an ion channel receptor protein designated PIEZO1 (Piezo1); the Greek word for Piesi, signify "pressure". Similarly, another receptor protein (ion channel) PIEZO2 (Piezo2), is located on the cell membrane, which too is sensitive towards external stress (Fig. 2). Further ectopic expression of Piezol on human embryonic kidney cells (HEK-293) translates these cells to be mechanosensitive (Coste et al., 2010). Several other mechanosensitive genes have been identified in vertebrate species, yet the results of their functional experiments are inconclusive. Mechanosensitive ion channels are specialized for the transduction of mechanical force directly activated by stresses to the lipid bilayer or its associated nonmembrane components on cells. Gene deletion experiments indicated that the elimination of the Piezo2 gene during embryonic development could cause respiratory distress and death at birth. Deep dive analysis of these experiments indicated that these PIEZO2 ion channels are located on pulmonary stretch receptors in the wall of bronchi, and bronchioles are subject to activation during the act of stretching while inhalation and the reflex of this stretching protect the lungs from over-inflation (Nonomura et al., 2017). Along with collaborators, Patapoutian 
demonstrated that baroreflex of arteries that monitor and regulate the blood pressure relies on PIEZO1 and PIEZO2, whereas deletion of these genes in mice can escalate the blood pressure to an extreme level as recorded in humans with baroreflex failure (Zeng et al., 2018). Enterochromaffin cells secrete hormones, and paracrine signalling molecules in response to mechanical stimulation generated due to the presence of gastrointestinal luminal content are known to be inherently mechanosensitive due to the presence of PIEZO1 and PIEZO2 gene (Wang et al., 2017). Similarly, sensory neurons present in urothelial cells and bladder having PIEZO1 and PIEZO2 exhibit sensitivity to bladder pressure in the absence of these two genes, there is impaired urinary bladder control (Marshall et al., 2020).

\section{The hope of rays for better days}

The responsibility of sensing the difference

\section{REFERENCES}

Caterina MJ, Schumacher MA, Tominaga M, Rosen TA, Levine JD et al., 1997. The capsaicin receptor: A heatactivated ion channel in the pain pathway. Nature, 389(6653): 816-824, doi: 10.1038/39807

Coste B, Mathur J, Schmidt M, Earley TJ, Ranade S et al., 2010. Piezo1 and Piezo2 are essential components of distinct mechanically activated cation channels. Science, 330(6000): 55-60, doi: 10.1126/ science. 1193270

DeLeo JA, 2006. Basic science of pain. J Bone Joint Surg Am, 88(Suppl 2): 58-62, doi: 10.2106/ JBJS.E.01286

Marshall KL, Saade D, Ghitani N, Coombs AM, Szczot $\mathrm{M}$ et al., 2020. PIEZO2 in sensory neurons and urothelial cells coordinates urination. Nature, 588(7837): 290-295, doi: 10.1038/s41586-020-28307

Martinac B, Buechner M, Delcour AH, Adler J and Kung C, 1987. Pressure-sensitive ion channel in Escherichia coli. Proc Natl Acad Sci USA, 84(8): 2297-2301, doi: 10.1073/pnas.84.8.2297

Moayedi M and Davis KD, 2013. Theories of pain: from specificity to gate control. J Neurophysiol, 109(1): 512, doi: 10.1152/jn.00457.2012

Nonomura K, Woo SH, Chang RB, Gillich A, Qiu Z between the heat, cold, and pressure of our environment depends on the nerve cells. As these cells are sensitive, they respond to us by accurately estimating the physical world. Frequent exposure to capsaicin results in a relatively low response to sensitive nerve cells. All this information indicates that these receptors can sense the heat and cold as well as the state of motion and balance of the body and acts accordingly. The compression and diffusion of the bladder are controlled by this ion channel while keeping the blood pressure under control. If we are unable to feel the temperature, we may inadvertently damage the cells and organs of our body by touching deadly hot and bitterly cold objects. Therefore, the pain response protects us from noxious heat and cold. On the other hand, chronic pain causes agony. We can hope that with this discovery in the future, the medical fraternity will be able to alleviate the pain of patients.

et al., 2017. Piezo2 senses airway stretch and mediates lung inflation induced apnoea. Nature, 541(7636): 176181, doi: 10.1038/nature20793

Peier AM, Moqrich A, Hergarden AC, Reeve AJ, Andersson DA et al., 2002. TRP channel that senses cold stimuli and menthol. Cell, 108: 705-715, doi: 10.1016/s0092-8674(02)00652-9

Stevens JC and Choo KK, 1998. Temperature sensitivity of the body surface over the life span. Somatosens Mot Res, 15(1): 13-28, doi: 10.1080/08990229870925

Stranding S, 2016. A brief history of topographical anatomy. J Anat, 229(1): 32-62, doi: 10.1111/joa.12473 Wang F, Knutson K, Alcaino C, Linden DR, Gibbons SJ et al., 2017. Mechanosensitive ion channel Piezo2 is important for enterochromaffin cell response to mechanical forces. J Physiol, 595(1): 79-91, doi: $10.1113 / \mathrm{JP} 272718$

Wood JN, Winter J, James IF, Rang HP, Yeats J et al., 1988. Capsaicin-induced ion fluxes in dorsal root ganglion cells in culture. J Neurosci, 8(9): 3208-3220, doi: 10.1523/JNEUROSCI.08-09-03208.1988

Zeng WZ, Marshall KL, Min S, Daou I, Chapleau MW et al., 2018. PIEZOs mediate neuronal sensing of blood pressure and the baroreceptor reflex. Science, 362(6413): 464-467, doi: 10.1126/science.aau6324

Received - 28.10.2021, Accepted - 23.11.2021, Published - 01.12.2021 